\title{
Sekundäre Linsenimplantation: chirurgische Techniken und Ergebnisse
}

\author{
Claudette Abela-Formanek (D) - Adrian Reumüller
}

Eingegangen: 7. April 2020 / Angenommen: 7. Juli 2020 / Online publiziert: 25. August 2020

(C) Der/die Autor(en) 2020

\begin{abstract}
Zusammenfassung Es stehen verschiedene alternative chirurgische Optionen zur Verfügung, um eine sekundäre Intraokularlinse (IOL) in Augen mit unzureichender Kapselunterstützung zu implantieren. Erfolgreiche Techniken umfassen die Implantation einer irisfixierten IOL (IFIOL), einer kammerwinkelgestützten Vorderkammerlinse (ACIOL) oder verschiedene Variationen zur Sklerafixierung (SFIOL). Jede dieser Methoden hat sich als sicher und effektiv erwiesen, weist jedoch verfahrensspezifische Einschränkungen auf. Jüngste Studien zeigen, dass die Entwicklung neuer chirurgischer Techniken diesen Patienten weiterhin sichere und reproduzierbare Behandlungsmöglichkeiten bietet. Obwohl die Implantation von sekundären IOLs ohne Kapselunterstützung von vielen Faktoren, einschließlich der Präferenz des Chirurgen, abhängt, sind nahtlose sklerafixierende Techniken für die chirurgische Gemeinschaft von wachsendem Interesse. Das Bestreben nach kürzeren und weniger traumatischen Operationen sowie nach reproduzierbaren und funktionell guten Ergebnissen, fördert dabei die Entwicklung von neuen Operationstechniken, Intraokularlinsen und Instrumenten. Diese Übersichtsarbeit gibt einen Einblick in alte und neue Behandlungsmethoden zur Korrektur von Aphakie mit sekundären Intraokularlinsen
\end{abstract}

Schlüsselwörter Sekundäre IOL-Implantation · Sklerale Fixierung · Nahtlose intrasklerale Fixierung • Irisfixierte IOL · Aphakie · Späte spontane IOL-Dislokation

\section{Secondary lens implantation: surgical techniques and outcome}

Summary Several alternative surgical options are available to implant a secondary intraocular lens (IOL) in eyes with inadequate capsular support. Successful techniques include placement of an iris-fixated IOL (IFIOL), chamber angle-supported anterior chamber IOL (ACIOL) or various approaches to scleral fixation (SFIOL) of a posterior lens. Each of these methods has been found to be safe and effective but show procedure-specific limitations. Recent studies have shown that the development of new surgical techniques continues to provide safer and reproducible treatment options for these patients. Although the implantation of secondary IOLs in the absence of capsular support depends on many factors including the surgeons preference, sutureless scleral fixation techniques are of immense growing interest to the surgical community. Shorter surgery time, reproducible and less traumatic techniques with a reduction in suture-related complications, and good functional outcomes are reasons for the development of new surgical techniques, intraocular lenses and instrumentation. This review summarizes old and new treatment practices for the correction of vision with secondary IOLs in the aphakic setting.

Keywords Secondary IOL implantation · Scleral fixation · Sutureless intrascleral fixation · Iris-fixated IOL $\cdot$ Aphakia $\cdot$ Late spontaneous in-the-bag IOL dislocation

Historisch betrachtet, waren Aphakie und Linsenluxationen die wichtigsten Indikationen zur sekundären Implantation einer Kunstlinse (intraokulare Linse [IOL]). Durch die höhere Lebenserwartung sowie 
durch komplikationsärmere Phakoemulsifikationstechniken, aber auch durch die Zunahme an phakorefraktiven Eingriffen steigt die Zahl der Menschen mit Pseudophakie kontinuierlich. Durch diese Entwicklung ist es wahrscheinlich, dass auch späte Inthe-bag-IOL-Dislokationen an Häufigkeit zunehmen. Solche Dislokationen nach Kataraktoperationen sind ernst zu nehmende Herausforderungen für Ophthalmochirurgen. Die Notwendigkeit einer Re-Operation aufgrund einer späten In-the-bag-IOL-Dislokationen liegt bei $0,032-0,6 \%$ [1-3].

Die anatomische Ursache von späten Dislokationen liegt in einer progressiven Dehiszenz der Zonulafasern viele Jahre nach Kataraktoperationen ohne primäre Komplikation. Risikofaktoren sind eine herabgesetzte Stabilität des Aufhängeapparates im Rahmen eines Pseudoexfoliationssyndroms (PEX), einer Bindegewebsschwäche (z. B. Marfan-Syndrom) oder bei hoher Myopie sowie ein Zustand nach okulärem Trauma, insbesondere in Kombination mit vitreoretinalen Eingriffen, aber auch wiederholten Uveitiden und Retinitis pigmentosa [4-6]. Das Auftreten von spontanen IOL-Dislokationen erfolgt meist lange nach der Kataraktoperation mit einem mittleren Intervall von 6,9 bis 8,5 Jahren [3, 7, 8]. Das Risiko steigt mit zunehmender Zeit nach der Kataraktoperation und wurde in einer rezenten Studie für 5, 10, 15, 20 bzw. 25 Jahre nach Phakoemulsifikation mit $0,1 \%, 0,1 \%, 0,2 \%, 0,7 \%$ und $1,7 \%$ angeführt [9].

Das chirurgische Management von sekundären IOL-Dislokationen ist komplex und kann mit zusätzlichen Komplikationen verbunden sein: Neben der operativen Repositionierung ist häufig auch eine Explantation der IOL mit dem gesamten Kapselsack erforderlich. Die fehlende adäquate Stabilität der vorderen oder hinteren Kapsel erfordert zudem den Einsatz von speziellen Operationstechniken. $\mathrm{Zu}$ den derzeit verfügbaren Techniken zählen vorderkammergestützte IOL („anterior chamber intraocular lens“ [ACIOL]), irisfixierte IOL (IFIOL) und sklerafixierte IOL (SFIOL). Ein umfassendes Review über IOLImplantationen bei fehlender Kapselstabilität wurde 2003 von der American Academy of Ophthalmology durchgeführt [10]. Die Autoren verglichen das Ergebnis und die Komplikationsrate zwischen ACIOL, IFIOL und SFIOL. Die damals zur Verfügung stehende Literatur kam zum Schluss, dass keine ausreichende Evidenz vorlag, um eine der 3 Methoden bei jenen Patienten zu favorisieren, bei denen alle 3 Techniken möglich gewesen wären [11-13]. Auch rezenteren Studien gelang es nicht, einen signifikanten Unterschied in postoperativer bester korrigierter Sehstärke („best corrected visual acuity“ [BCVA]) oder Komplikationsraten zwischen ACIOL, IFIOL und SFIOL zu zeigen [14-17]. Späte IOL-Dislokationen stellen den Operateur daher vor die Herausforderung, den geeigneten chirurgischen Zugang und die ideale Position der IOL zu finden. Jede Operationstechnik hat Vor- und Nachteile, die vorsichtig abgewogen werden müssen. Diese
Übersichtsarbeit hat zum Ziel, die aktuellen Daten und Techniken für sekundäre IOL-Implantationen aufzuzeigen.

\section{Präoperative Überlegungen}

Verschiedene Faktoren des individuellen Patienten müssen bei der Evaluation der geeigneten Operationstechnik berücksichtigt werden. Ein wesentlicher Faktor ist das Alter des Patienten, und die meisten Chirurgen empfehlen, auf einen Einsatz von ACIOL bei jungen Patienten aufgrund der potenziellen chronischen Schädigung des Hornhautendothels zu verzichten. Bei Patienten, die bereits präoperativ eine geringere Endothelzelldichte aufweisen, oder bei Patienten nach Hornhauttransplantationen empfiehlt sich prinzipiell eine IOL-Implantation hinter der Iris. Multimorbide Patienten und im Speziellen jene mit erforderlicher antikoagulativer Therapie profitieren von kürzeren Operationszeiten und geringerer chirurgischer Manipulation durch weniger invasive Operationstechniken. Patienten mit Irisdefekten oder Irisatrophie sind für irisfixierte Verfahren denkbar wenig geeignet. Ähnlich verhält es sich mit sklerafixierenden Techniken bei Vorliegen von skleralen Ausdünnungen und Erkrankungen, beispielsweise nach multiplen Voroperationen, bei Skleromalazie, ausgeprägter Myopie oder bei Störungen des Kollagenstoffwechsels.

Auch bezüglich einer Explantation der alten IOL müssen mehrere Faktoren berücksichtigt werden. Selbst wenn eine Reststabilität des Kapselsackkomplexes vorliegt, sollten Single-Piece-IOL aufgrund ihrer dicken Haptik nicht im Sulkus positioniert werden, da dies zu Erodieren der Iris, sekundären Glaskörperblutungen oder dem Uveitis-Glaukom-HyphämaSyndrom (UGH-Syndrom) führen kann [18]. Subluxierte Single-Piece-IOL mit intaktem Kapselkomplex können allerdings mittels Skleranähten fixiert werden. In den Glaskörper luxierte Single-Piece-IOL müssen prinzipiell explantiert werden.

Ältere Patienten oder hochmyope Patienten haben ein höheres Risiko für choroidale Effusionssyndrome, deren Auftreten mit größeren Inzisionen und intraoperativ auftretender okulärer Hypotonie verbunden ist. Kleinschnittchirurgie sowie der Einsatz von Kanülen zur Aufrechterhaltung der Vorderkammer (beispielsweise ein „anterior chamber maintainer“ nach Lewicky) oder der Einsatz von Pars-plana-Infusionskanülen können dem Auftreten dieser schwerwiegenden Komplikation entgegenwirken.

Durch das mögliche Auftreten von unvorhergesehenen intraoperativen Komplikationen wie IOL-Dislokationen in den Vitreus, Netzhautdefekten oder Netzhautabhebungen ist es von Vorteil, wenn diese herausfordernden Eingriffe von Operateuren mit Erfahrung in vitreoretinaler Chirurgie durchgeführt werden. Der Einsatz von modernen vitreoretinalen Instrumenten erlaubt oftmals die Bergung der IOL und im geeigneten Fall die Fixierung an die Iris oder die Sklera. 
Ein grundsätzliches Problem stellt der postoperative refraktive Fehler dar, da die endgültige effektive Position der IOL nicht exakt bestimmbar ist. Bei der Refixation einer vorhandenen IOL muss die stärkere Wirkung der Brechkraft bei irisnaher Positionierung berücksichtigt werden. Beim Austausch von Linsen muss die Brechkraft der eingesetzten IOL abgeschwächt werden, um bei anteriorem Sitz eine ähnliche Zielrefraktion zu erhalten wie bei Implantation in den Kapselsack [19].

\section{Chirurgische Techniken}

\section{Vorderkammergestützte Linse (ACIOL)}

Die möglichen Indikationen für den Einsatz von ACIOL stellen eine Instabilität des Kapselkomplexes sowie das Vorbestehen von generellen Komorbiditäten insbesondere bei älteren Patienten mit einer reduzierten Lebenserwartung dar. Die Operationstechnik umfasst eine vordere Vitrektomie, eine $7-8 \mathrm{~mm}$ lange Peritomie sowie das Anlegen eines 6,5-7 mm langen Skleratunnels und die anschließende Implantation der Linse. Die IOL-Haptiken werden im iridokornealen Winkel positioniert und die Wunde anschließend mit Nylon verschlossen.

Aufgrund der individuellen Vorderkammertiefe und des Hornhautdurchmessers sind ACIOL mit einem höheren Risiko von Hornhautödemen assoziiert. Ist die ACIOL zu klein, ist ihre Verankerung im Kammerwinkel oftmals unzureichend, wodurch die Entstehung von traumatischen Endothelschäden begünstigt wird. Ist die ACIOL zu groß, kann sie zur chronischen Vernarbung des Kammerwinkels, intraokulärer Entzündung und schließlich zu einem Kammerwinkelverschluss führen. Ein im Jahr 2015 von Chan et al. publizierter Vergleich zwischen einer primären ACIOL- und sekundären SFIOL-Implantation zeigte allerdings keine Langzeitunterschiede im visuellen Ergebnis und dem Komplikationsprofil bei Patienten mit fehlender Stabilität des Kapselkomplexes [14, 20].

\section{Irisfixierte Linse (IFIOL)}

Irisklauenlinsen sind in Europa, Kanada und Südamerika weit verbreitet. Die Artisan Aphakic IOL (Ophthec ${ }^{\circledR}$ BV, Gröningen, The Netherlands), in den USA unter dem Namen Verisyse ${ }^{\circledR}$ geführt (Johnson \& Johnson), ist eine Weiterentwicklung der von Worst entworfenen „Worst Iris Claw IOL“ [21]. Operationstechnisch wird nach einer vorderen Vitrektomie, präferenziell einer anterioren Pars-plana-Vitrektomie, die Iris durch Gabe eines Miotikums verengt und anschließend die IOL mittels ihrer Klauenhaptiken im Bereich der mittelperipheren Iris verankert, um möglichst wenig Reizung in der Vorderkammer zu verursachen [22]. Entsprechend älteren Daten ist der prä- und retropupilläre Einsatz von IFIOL auch bei Patienten nach penetrierender Keratoplastik (PKP)

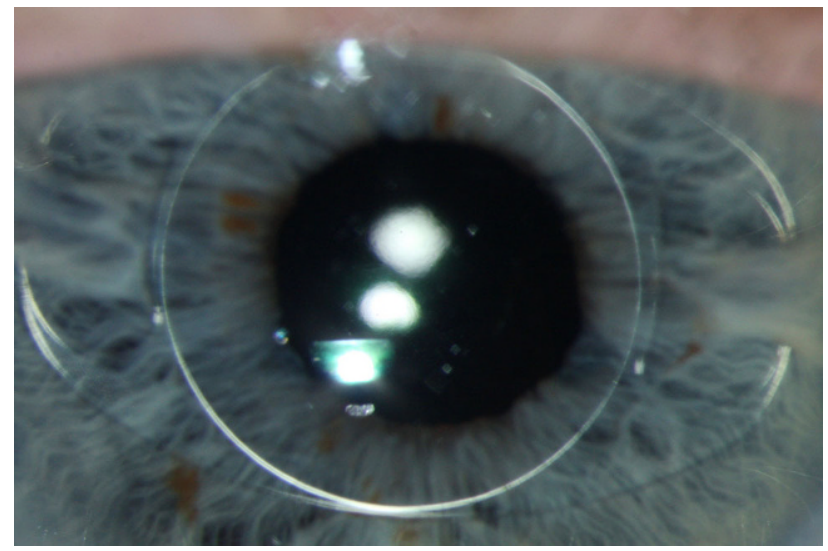

Abb. 1 Präpupilläre irisfixierte IOL

möglich, um eine Pseudophakie bei unzureichender Kapselstabilität zu erreichen [23]. In Augen nach PKP zeigt die retropupilläre Technik verglichen mit der präpupillären Implantation eine tiefere Vorderkammer und einen weiter geöffneten Kammerwinkel [24].

Die optimale Platzierung der IFIOL wird fortlaufend diskutiert, und je nach Studie wird eine prä- oder retropupilläre Platzierung empfohlen [25]. Bezüglich der Endothelzelldichte zeigt sich bei retropupillärer Implantation auch nach über 5 Jahren kein statistisch signifikanter Abfall [26, 27]. In einem rezenten Vergleich von 87 Augen mit präpupillärer und 93 Augen mit retropupillärer IFIOL-Fixation zeigte sich kein statistisch signifikanter Unterschied in der Endothelzelldichte nach 5 Jahren (Abb. 1 und 2). Andere Autoren beschreiben eine vergleichbare Reduktion der Endothelzelldichte von 9-10\% bei präpupillärer Implantation [28, 29] und $8-13 \%$ bei retropupillärer Implantation [30, 31]. Fünf-Jahres-Daten zeigen, dass sowohl die prä- als auch die retropupilläre sekundäre Implantation von IFIOL ein gutes Ergebnis und eine hohe Sicherheit bei Patienten mit fehlender Kapselkomplexstabilität aufweisen [32, 33].

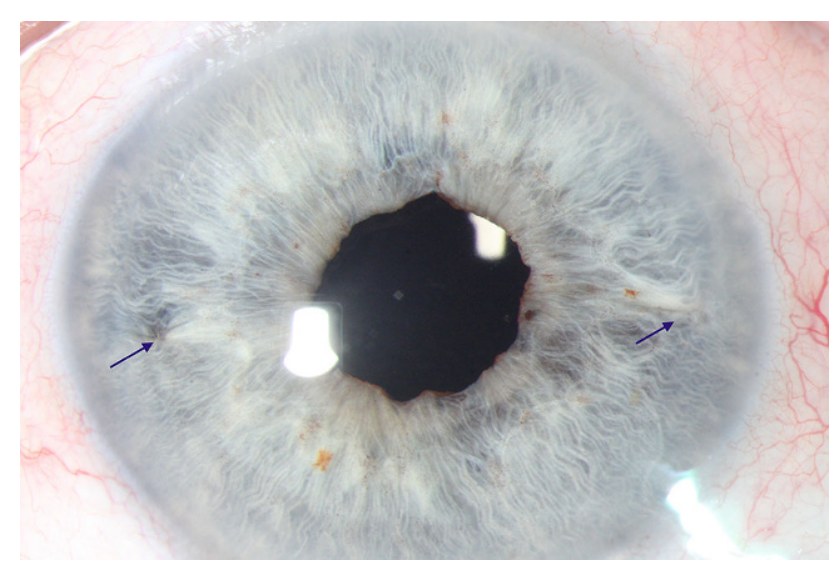

Abb. 2 Retropupilläre irisfixierte IOL. Pfeile zeigen auf die Stelle der retropupillären Enklavation 
Die häufigste postoperative Komplikation einer IFIOL ist die spontane Deenklavation, die bei $1-3 \%$ der Patienten auftritt $[25,26,28,32,34]$. Ein weiterer Nachteil besteht in der Notwendigkeit einer 5,5mm-Inzision und der Induktion eines postoperativen Astigmatismus. Baykara et al. beschrieben die Technik eines skleralen, nahtlosen Tunnels [35], mit dem erfahrene Chirurgen einen mittleren chirurgisch induzierten Astigmatismus von ca. 1 dpt erzielten $[33,35]$. Das Auftreten eines zystoiden Makulaödems ist ebenfalls eine relative häufige Komplikation, die zwischen 2 und 12\% der sekundären IFIOL-Implantationen nach vorangegangener IOL-Dislokation auftritt $[5,7,32,34,36,37]$.

Alternativ zu Irisklauenlinsen kann eine irisgenähte Fixierung der IOL mit modifizierter McCannel-Naht oder bevorzugter Weise mit einem Siepser-Knoten in Betracht gezogen werden. Während der IOL-KapselKomplex mit einem zweiten Instrument vorübergehend in Position gehalten wird, wird eine 10-0-Polyprolennaht auf einer langen gekrümmten Nadel durch eine Hornhautparazentese geführt, um die periphere Iris sowie die fibrotische Kapsel und die IOL-Haptik $\mathrm{zu}$ fassen. Diese Technik ist besonders hilfreich bei leichten, unkomplizierten IOL-Subluxationen, bei denen lediglich eine geringe IOL-Reposition erforderlich ist. Pupillenovalisation und CME, „iris-optic-capture“ sowie eine Pigmentdispersion stellen häufige Komplikationen dar. Um einer Glaskörperinkarzeration oder -traktion am Ende der Operation vorzubeugen, ist nach der Irisnahtfixierung eine sorgfältige Vitrektomie empfehlenswert.

\section{Transsklerale nahtfixierte Linsen}

In den 1980ern haben Malbran et al. erstmals die genähte Fixation einer Kunstlinse bei Aphakie nach intrakapsulärer Kataraktextraktion beschrieben [38]. Die Operationstechnik umfasste die Nahtfixation der Haptiken bei 3 und 9 Uhr mittels eines 10-0-Polypropylenfadens, der in einer Distanz von $2 \mathrm{~mm}$ hinter dem Limbus geführt wurde. Hierfür wurde eine 28-GaugeNadel ab externo eingebracht, um bei beiden Uhrzeiten eine intraokuläre Schleife aus dem Nahtmaterial zu formen. Über eine korneale Inzision wurden diese Schleifen externalisiert und die Haptiken mittels eines Ankerknotens befestigt. Anschließend wurde durch Zug an den Fäden die IOL ins Auge gebracht, hinter der Iris positioniert, und die Fäden wurden an die Sklera geknotet. Im Jahr 1991 präsentierte Lewis die Nahtdeckung mittels Skleralappen, um einem Erodieren der Konjunktiva durch die Fäden vorzubeugen [39]. Zusätzlich benutzte er eine gerade Nadel an einem 10-0-Polypropylenfaden, die er mit der Spitze voran in der Kanülenöffnung einer $180^{\circ}$ gegenüberliegenden 28-Gauge-Nadel „andockte“. Beide Nadeln wurden $2 \mathrm{~mm}$ hinter dem posterioren Limbus ins Auge gebracht. Diese 2-Nadel-Technik führte zu einer besser reproduzierbaren Positionierung der Nähte und damit zu einer verlässlicheren Implantation der IOL im Bereich des Sulcus. Auch heute wird diese Technik der Nahfixation mit guten Erfolgsraten eingesetzt, und eine kleine Studie im Jahr 2015 zeigte 5 bis 10 Jahre nach der Operation bei lediglich 2 von 13 Augen eine geringe Linsendezentrierung ohne relevanten Einfluss auf den Visus [40].

Seit der Einführung der skleralen Nahtfixierung von IOL wurden das Material und die Technik modifiziert, um die Erfolgsraten zu erhöhen und die Komplikationsraten zu senken. Aufgrund von Fadenbruch durch Materialabbau werden 10-0-Polypropylenfäden zunehmend durch 9-0-Polypropylen oder 7-0-Gore-Tex (CV-8) ersetzt [41]. Ein größerer Nahtdurchmesser bedarf aber einer größeren Sklerotomie, womit das Risiko einer Leckage durch den Stichkanal erhöht wird. Neben dem technisch schwierigeren Handling von stärkerem Nahtmaterial werden auch die Knoten größer und erhöhen das Risiko von Bindehauterosionen, wenn sie nicht vollständig versenkt werden können.

Neben IOL-Dislokationen durch ein Verrutschen der Fäden im Bereich der Haptik ist die Zwei-PunktFixierung empfänglich für IOL-Verkippungen, die Aberrationen höherer Ordnung verursachen. In einem direkten Vergleich zwischen nahtfixierten IOL mit im Kapselsack platzierten IOL zeigte sich ein doppelt so großer Verkippungswinkel bei den genähten SFIOL $\left(6,35^{\circ}\right.$ vs. $\left.3,18^{\circ}\right)[42,43]$. Späte Dislokationen der genähten SFIOL entstehen häufig durch einen Fadenbruch des 10-0-Polypropylenmaterials [44], der mit einer Häufigkeit von 0,5 [45] bis $15 \%$ der genähten SFIOLs [44, 46] und am häufigsten in einem Zeitraum von 3 bis 9 Jahren (bis hin zu 24 Jahren) nach der Operation auftritt [45, 47].Langzeitergebnisse von anderen Materialen wie Gore-Tex sind noch ausständig. Zumindest zeigte eine Studie von 85 Augen mit 7-0-Gore-Tex keinen Fall eines Fadenbruchs während eines kürzeren Follow-up von 3 bis zu 33 Monaten [41].

Modifikationen der Nahtfixationstechnik beinhalten die Internalisierung der Knoten, das Anlegen von Sklerotomien unter dem Skleralappen und von korneoskleralen Taschen (Hoffman-Pockets), um den Knoten innerhalb der Sklera zu versenken und die Bindehaut vor möglichem Erodieren zu schützen. Eine weitere Methode stellt die „Z-Naht“ dar, bei der das externalisierte Nahtmaterial in einem Zickzackverlauf 5-mal innerhalb der Sklera verankert wird. In einer Serie von 45 IFIOL mittels Z-Naht-Technik (10-0-Polypropylen) wurde in einem Follow-up von 6 bis zu 38 Monaten kein Fall von IOL-Dislokationen festgestellt [48].

Der Einsatz von sämtlichen Nahtfixationstechniken ist allerdings technisch herausfordernd und bedingt eine anteriore bzw. im Optimalfall eine Pars-planaVitrektomie, um das Risiko von traktionsbedingten Netzhautrissen zu vermeiden [49]. Auch darf nicht übersehen werden, dass das wiederholte Penetrieren 
der Sklera und der Uvea mit der Nadel das Risiko von suprachoroidalen Blutungen erhöht. Ein besonderes intra- und postoperatives Blutungsrisiko haben Patienten unter laufender Antikoagulation. Neben IOLVerkippungen und Dislokationen sind auch späte Infektionen möglich. Langfristig sind zudem Komplikationen wie postoperative Hypotonie, aber auch Fälle von Hypertonie, Binderhauterosion trotz Anlegen eines Skleralappens sowie das Auftreten von CME zwischen 1 und $12 \%$ der Fälle beschrieben [50, 51].

\section{Intrasklerale nahtlose skleral fixierte IOL}

Die nahtassoziierten Nachteile der sklerafixierten Operationstechniken führten $\mathrm{zu}$ Überlegungen, ob eine sekundäre sklerale IOL-Fixation auch ohne Nahtmaterial durchführbar wäre. Die erste Beschreibung einer nahtlosen skleralen IOL-Fixation gelang Scharioth et al., indem 2 gegenüberliegende Sklerotomien in einem posteriolimbalen Abstand von 1,5-2 $\mathrm{mm}$ mittels 24-Gauge-Nadeln angelegt wurden. Ausgehend davon, wurden im nächsten Schritt 2 limbusparallele sklerale Tunnelierungen gesetzt. Eine 3-stückige-IOL wurde anschließend ins Auge eingebracht, die Haptiken durch die Sklerotomien externalisiert und in den Tunnelierungen versenkt $[52,53]$.

Einen etwas aufwendigeren Zugang wählten Agarwal et al., die Skleralappen und Fibrinklebstoff zur nahtlosen Fixation kombinierten [54]. Hierfür wurden zuerst zwei $180^{\circ}$ gegenüberliegende, limbusbasierte Lappen mit halber Skleradicke angelegt. Mit einer 22-Gauge-Nadel wurden anschließend 2 Sklerotomien unter den Lappen angelegt und die Haptiken durch diese externalisiert. Anschließend wurden die Haptiken mittels Fibrinklebstoff am Sklerabett befestigt. Während diese Technik auch nach 5 Jahren noch eine gute Positionierung der IOL zeigte, ist die Präparation der beiden Skleralappen aufwendig und benötigt chirurgisches Geschick [55].

Das Prinzip der Externalisierung der Haptiken über Sklerotomien wurden in den Folgejahren wiederholt modifiziert. Insbesondere der Einsatz von vitreoretinalen Instrumenten wie der Skleraltunnelanlage mittels 23-G-, 25-G- und 27-G-Trochar oder die Externalisierung der Haptik mittels vitreoretinaler Pinzette wurde in den Folgejahren getestet, wobei insbesondere der Einsatz von 27-G-Trocharen zu relativ dislokationsresistenten IOL-Positionen führte [19, 56-58].

Einen besonders geradlinigen Zugang („double needle flanged technique“) beschrieben Yamane et al., die mittels einer transkonjunktivalen Stichinzision mit zwei 27-Gauge-Nadeln 2 gegenüberliegende Sklerotomien im Abstand von $2 \mathrm{~mm}$ hinter dem Limbus anlegten [59]. Im nächsten Schritt wurden die Haptiken einer in der Vorderkammer positionierten, 3-stückigen IOL mit einer Mikropinzette gefasst und in den Kanülenöffnungen der 27-Gauge-Nadeln versenkt. Anschließend wurden beide Nadeln zeitgleich aus der Sklera gezogen und die Haptiken so externalisiert. Die

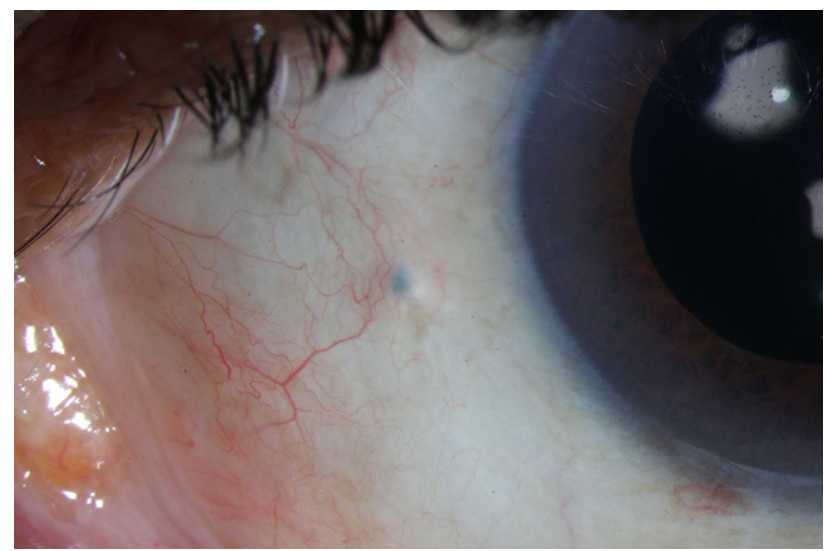

Abb. 3 Subkonjunktivale reizfreie Haptikenden („flange“) nach der Implantationstechnik von Yamane

freiliegenden, langen Haptiken wurden anschließend mittels Kaustik zu einem kleinen Knoten („flange“) verkürzt und verschmolzen. Dieser Knoten verankert die Linsenhaptik in dem Sklerotomietunnel (Abb. 3). Eine pars plana Vitrektomie ist zwingend erforderlich. Bei 100 Augen fanden die Autoren nach 20 Monaten keine schweren Komplikationen. Zu den postoperativen Komplikationen gehörten der „iris-optic capture“ (8\%) (Abb. 4), Glaskörperblutungen (5\%), frühe postoperative Hypotonien (2\%), frühe okuläre Hypertonien von mehr als $25 \mathrm{mmHg}(2 \%)$ und das zystoide Makulaödem (1\%). Der Hornhautendothelzellverlust betrug $1,2 \%$ nach 6 Monaten, 4,3\% nach 12 Monaten und 6,5\% nach 24 Monaten. Zudem zeigte sich eine klinisch nicht relevante mittlere IOL-Verkippung von $3,4^{\circ}$.

Der Vorteil dieses Zuganges besteht darin, dass weder die Bindehaut großflächig eröffnet werden muss, noch ist eine ausgeprägte Manipulation der Sklera erforderlich [60, 61]. Allerdings benötigt das Einführen der zweiten Haptik in die 27-Gauge- oder 30-GaugeNadel ein hohes Maß an Geduld und Geschick. Bonnell et al. beschrieben eine modifizierte Methode, wobei die 2. Haptik zunächst in den Glaskörperraum ein-

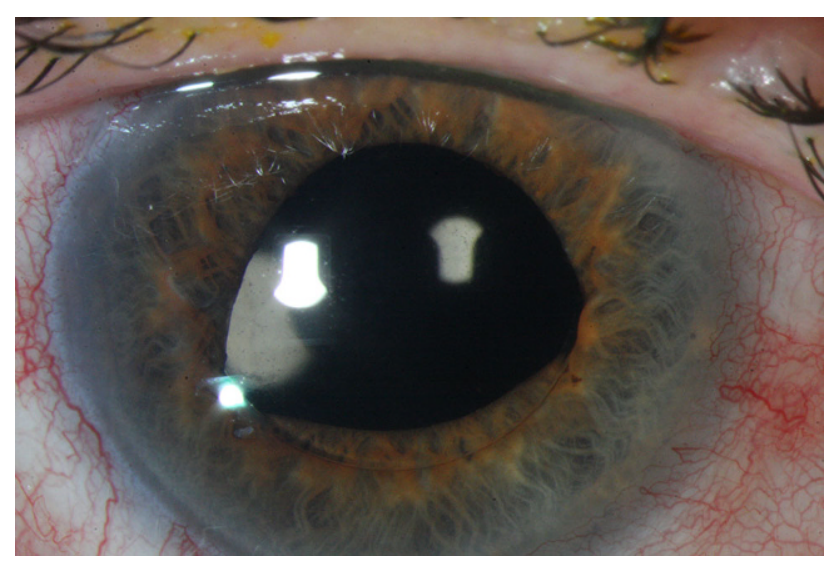

Abb. 4 „Iris optic capture“ nach intraskleraler IOL-Implantation nach Yamane 
geführt wurde, um dann in die zweite 27-Gauge-Nadel eingeführt zu werden [62].

Bei dieser Technik ist die Schaffung symmetrischer intraskleraler Tunnel auf beiden Seiten des Limbus eine Herausforderung. Winkel und Länge des intraskleralen Teils der Nadelpassage beeinflussen die IOLNeigung und die optische Zentrierung erheblich. Ferner kann ein zu kleiner „flange“ zu einer postoperativen IOL-Dislokation führen, während ein zu großer „flange“ schwierig in den Skleratunnel zu drücken ist. Zur kontrollierteren Formung des Flange-Durchmessers können die Haptikenden vor der endgültigen Kauterisation nach Bedarf gekürzt werden.

Unlängst wurde die Technik von Canabrava et al. modifiziert. Dabei wurden die Kapselspannringe bis hin zu vierflügeligen IOL mittels 5,0-Prolene transkonjunktival und transskleral fixiert. Anschließend wurden die losen Fäden gekürzt, mittels Kaustik zu einem „flange“ geformt und intraskleral fixiert [63, 64]. Als Weiterentwicklung der intraskleralen nahtlosen Technik wurde unlängst eine einteilige, hydrophile Intraokularlinse (FIL SSF Carlevale IOL, Soleko, IOL Division, Italien) eingeführt.

Nach vorausgegangener pars plana Vitrektomie werden die Ankerförmigen Haptiken unter zwei gegenüberliegenden Skleralappen fixiert. Die derzeitigen Ergebnisse sind vielversprechend, und die Linse wird eine Ergänzung in der zukünftigen Diskussion der sekundären IOL Implantation darstellen [65].

\section{Genähte vs. nahtlose skleral fixierte IOLs}

Es gibt kaum Studien, die verschiedene SFIOL-Techniken direkt gegenüberstellen. Ganekal et al. verglichen nahtfixierte SFIOL-Implantationen $(n=25)$ mit Fibrinklebstoff-assistierter SFIOL-Implantation $(n=25)$ und fanden in beiden Gruppen einen vergleichbaren postoperativen Visus. Allerdings zeigten sich in der nahtfixierten Gruppe mehr Komplikationen als in der Fibrinklebstoff-assistierten (56\% vs. $28 \%, p=0,045$ ), wie beispielsweise das Auftreten von Entzündungen oder postoperativer okulärer Hypertension [66]. Eine vergleichende Studie zur transskleralen nahtfixierten und intrasklerafixierten Intraokularlinsenimplantation mit nur 1-monatigem Follow-up zeigte eine IOLVerkippung und eine passagere Glaskörperblutung bei 4 von 45 Augen in der transskleralen nahtfixierten Gruppe und nur bei 2 von 44 Augen in der sklerafixierten Gruppe. Eine langfristige Nachuntersuchung wäre erforderlich, um die Stabilität des Nahtmaterials in der ersten Gruppe zu bewerten. Allerdings lassen die Dauer und die Patientenzahl der Studien keine verlässlichen Schlüsse bezüglich der definitiven Überlegenheit einer der beiden Techniken zu [61].

\section{Zusammenfassung}

Erworbene oder angeborene Schwächen der Zonulafasern durch Erkrankungen, okuläre Traumen oder komplexe Kataraktoperationen können die Stabilität der vorderen und/oder hinteren Linsenkapsel beeinträchtigen und damit zu einer Subluxation oder Luxation der IOL führen. In diesen Fällen empfiehlt es sich für die meisten Patienten, eine funktionelle IOL-Position wiederherzustellen und eine Aphakie zu vermeiden. Die sekundäre Implantation einer ACIOL, IFIOL oder SFIOL führt in vielen Fällen zum Wiedererlangen eines zufriedenstellenden Visus. Neben individuellen Risiken muss auch die Anatomie des Auges berücksichtigt werden. Bei Patienten mit unzureichender Kapselstabilität und Erkrankungen oder Schäden an der Iris sind SFIOLs die einzig mögliche Methode.

SFIOL-Techniken können grob in nahtfixierte oder nahtlose Methoden unterteilt werden. Nahtfixierte SFIOL-Techniken variieren in Nahtmaterial, IOLTyp, Technik der Nahtplatzierung und der Art und Weise, wie die Knoten gesetzt und verdeckt werden. Das Voranschreiten von nahtlosen Techniken, indem die Haptik über Sklerotomien, sklerale Tunnel oder Lappen fixiert wird, versucht die Nachteile des Nahtmaterials zu eliminieren. Allerdings können auch bei diesen Techniken die IOLs dislozieren. Zudem fehlt insbesondere bei nahtlosen Techniken eine ausreichende Langzeiterfahrung hinsichtlich IOL-Stabilität und dem Auftreten von späten Komplikationen.

Die chirurgischen Techniken für SFIOL werden kontinuierlich adaptiert und neue innovative Methoden getestet. Mit zunehmender Weiterentwicklung von Nahtmaterialien, Instrumenten und Kunstlinsendesigns wird auch in nächster Zeit eine Vielzahl von neuen Operationsmethoden veröffentlicht werden. Neben der Entwicklung von immer neueren Methoden werden aber v. a. aussagekräftige Langzeitstudien notwendig sein, die verschiedene Techniken direkt miteinander vergleichen. Letztendlich muss es das Ziel der Ophthalmochirurgen sein, unter der Vielzahl der zur Verfügung stehenden Techniken jene zu finden, die nicht nur innovativ sind, sondern auch langfristige stabile Ergebnisse liefern.

Trotz der oftmals guten Studienergebnisse der verschiedenen Techniken weisen wir auf den Umstand hin, dass Patienten einer ausreichenden Aufklärung über sekundäre Implantationstechniken bedürfen. Sekundäre Implantationstechniken gehen mit deutlich höheren Komplikationsrisiken und einem unsichereren postoperativen visuellen Ergebnis einher als standardmäßige Phakoemulsifikationen.

Funding Open access funding provided by Medical University of Vienna.

Interessenkonflikt C. Abela-Formanek und A. Reumüller geben an, dass kein Interessenkonflikt besteht. Die Autoren haben kein finanzielles Interesse an Materialien, die in diesem Artikel behandelt werden.

Open Access Dieser Artikel wird unter der Creative Commons Namensnennung 4.0 International Lizenz veröffentlicht, welche die Nutzung, Vervielfältigung, Bearbeitung, Verbreitung und Wiedergabe in jeglichem Medium und Format erlaubt, 
sofern Sie den/die ursprünglichen Autor(en) und die Quelle ordnungsgemäß nennen, einen Link zur Creative Commons Lizenz beifügen und angeben, ob Änderungen vorgenommen wurden.

Die in diesem Artikel enthaltenen Bilder und sonstiges Drittmaterial unterliegen ebenfalls der genannten Creative Commons Lizenz, sofern sich aus der Abbildungslegende nichts anderes ergibt. Sofern das betreffende Material nicht unter der genannten Creative Commons Lizenz steht und die betreffende Handlung nicht nach gesetzlichen Vorschriften erlaubt ist, ist für die oben aufgeführten Weiterverwendungen des Materials die Einwilligung des jeweiligen Rechteinhabers einzuholen.

Weitere Details zur Lizenz entnehmen Sie bitte der Lizenzinformation auf http://creativecommons.org/licenses/by/4. $0 /$ deed.de.

\section{Literatur}

1. Jakobsson G, Zetterberg M, Lundstrom M, Stenevi U, Grenmark R, Sundelin K. Late dislocation of in-the-bag and outof-thebagintraocular lenses: ocular and surgical characteristics and time to lens repositioning. J Cataract Refract Surg. 2010;36(10):1637-44.

2. Clark A, Morlet N, Ng JQ, Preen DB, Semmens JB. Whole population trends in complications of cataract surgery over 22 years in Western Australia. Ophthalmology. 2011;118(6):1055-61.

3. Monestam EI. Incidence of dislocation of intraocular lenses and pseudophakodonesis 10 years after cataract surgery. Ophthalmology. 2009;116(12):2315-20.

4. Jehan FS, Mamalis N, Crandall AS. Spontaneous late dislocation of intraocular lens within the capsular bag in pseudoexfoliation patients. Ophthalmology. 2001;108(10):1727-31.

5. Gross JG, Kokame GT, Weinberg DV, Dislocated In-The-Bag Intraocular Lens Study Group. In-the-bag intraocular lens dislocation. Am J Ophthalmol.2004;137(4):630-5.

6. Gimbel HV, Condon GP, Kohnen T, Olson RJ, Halkiadakis I. Late in-the-bag intraocular lens dislocation: incidence, prevention, and management. J Cataract Refract Surg. 2005;31(11):2193-204.

7. Lorente R, de Rojas V, Vazquez de Parga P, Moreno C, Landaluce ML, Dominguez R, et al. Management of late spontaneous in-the-bag intraocular lens dislocation: retrospective analysis of 45 cases. J Cataract Refract Surg. 2010;36(8):1270-82.

8. Davis D, Brubaker J, Espandar L, Stringham J, Crandall A, Werner L, et al. Late in-the-bag spontaneous intraocular lens dislocation: evaluation of 86 consecutive cases. Ophthalmology. 2009;116(4):664-70.

9. PueringerSL, Hodge DO, ErieJC. Risk oflateintraocularlens dislocation after cataract surgery, 1980-2009: a populationbased study. Am J Ophthalmol.2011;152(4):618-23.

10. Wagoner MD, Cox TA, Ariyasu RG, Jacobs DS, Karp CL. Intraocular lens implantation in the absence of capsular support: a report by the American Academy of Ophthalmology. Ophthalmology. 2003;110(4):840-59.

11. Bellucci R, PucciV, MorselliS, Bonomi L. Secondaryimplantation of angle-supported anterior chamber and scleralfixated posterior chamber intraocular lenses. J Cataract RefractSurg. 1996;22(2):247-52.

12. Davis RM, Best D, Gilbert GE. Comparison of intraocular lens fixation techniques performed during penetrating keratoplasty. Am J Ophthalmol. 1991;111(6):743-9.
13. Lyle WA, Jin JC. Secondary intraocular lens implantation: anterior chamber vs posterior chamber lenses. Ophthalmic Surg. 1993;24(6):375-81.

14. Chan TC, Lam JK, Jhanji V, Li EY. Comparison of outcomes of primary anterior chamber versus secondary scleral-fixated intraocular lens implantation in complicated cataract surgeries. Am JOphthalmol.2015;159(2):221-226.e2.

15. Kim KH, Kim WS. Comparison of clinical outcomes of iris fixation and scleral fixation as treatmentfor intraocularlens dislocation. Am JOphthalmol.2015;160(3):463-469.e1.

16. Melamud A, Topilow JS, Cai L, He X. Pars plana vitrectomy combined with either secondary scleral-fixated or anterior chamber Intraocular lens implantation. Am J Ophthalmol. 2016;168:177-82.

17. Hazar L, Kara N, Bozkurt E, Ozgurhan EB, Demirok A. Intraocular lens implantation procedures in aphakic eyes with insufficient capsular support associated with previous cataract surgery. J Refract Surg. 2013;29(10):685-91.

18. Chang DF, Masket S, Miller KM, Braga-Mele R, Little BC, Mamalis N, et al. Complications of sulcus placement of single-piece acrylic intraocular lenses: recommendations for backup IOL implantation following posterior capsule rupture. J Cataract Refract Surg. 2009;35(8):1445-58.

19. Abbey AM, Hussain RM, Shah AR, Faia LJ, Wolfe JD, Williams GA. Sutureless scleral fixation of intraocular lenses: outcomes of two approaches. The 2014 Yasuo Tano Memorial Lecture. Graefes Arch Clin Exp Ophthalmol. 2015;253(1):1-5.

20. Brunin G, Sajjad A, Kim EJ, Montes de Oca I, Weikert MP, Wang L, et al. Secondary intraocular lens implantation: Complication rates, visual acuity, and refractive outcomes. JCataract RefractSurg. 2017;43(3):369-76.

21. Worst JG. Iris claw lens. J Am Intraocul Implant Soc. 1980;6(2):166-7.

22. Dick HB, Augustin AJ. Lens implant selection with absence of capsular support. Curr Opin Ophthalmol. 2001;12(1):47-57.

23. Rijneveld WJ, Beekhuis WH, Hassman EF, Dellaert MM, Geerards AJ. Iris claw lens: anterior and posterior iris surface fixation in the absence of capsular support during penetrating keratoplasty. J Refract Corneal Surg. 1994;10(1):14-9.

24. Gicquel JJ, Guigou S, Bejjani RA, Briat B, Ellies P, Dighiero P. Ultrasound biomicroscopy study of the verisyse aphakic intraocular lens combined with penetrating keratoplasty in pseudophakic bullous keratopathy. J Cataract Refract Surg. 2007;33(3):455-64.

25. Gonnermann J, Klamann MK, Maier AK, Rjasanow J, Joussen AM, Bertelmann E, et al. Visual outcome and complications after posterior iris-claw aphakic intraocular lens implantation. J Cataract Refract Surg. 2012;38(12):2139-43.

26. Forlini M, Soliman W, Bratu A, Rossini P, Cavallini GM, Forlini C. Long-term follow-up of retropupillary iris-claw intraocular lens implantation: a retrospective analysis. BMC Ophthalmol.2015;15:143.

27. Gonnermann J, Maier AK, Klamann MK, Brockmann T, Bertelmann E, Joussen AM, et al. Posterior iris-claw aphakic intraocularlensimplantation and descemet membrane endothelial keratoplasty. BrJ Ophthalmol.2014;98(9):1291-5.

28. Guell JL, Velasco F, Malecaze F, Vazquez M, Gris O, Manero F. Secondary artisan-verysise aphakic lens implantation. J Cataract Refract Surg. 2005;31(12):2266-71.

29. Chen Y, Liu Q, Xue C, Huang Z, Chen Y. Three-year follow-up of secondary anterior iris fixation of an aphakic intraocular lens to correct aphakia. J Cataract Refract Surg. 2012;38(9):1595-601. 
30. Ganesh S, Brar S, Relekar K. Long term clinical and visual outcomes of retrofixated Iris claw lenses implantation in complicated cases. Open Ophthalmol J. 2016;10:111-8.

31. Anbari A, Lake DB. Posteriorly enclavated iris claw intraocular lens for aphakia: long-term corneal endothelial safety study. Eur J Ophthalmol.2015;25(3):208-13.

32. Toro MD, Longo A, Avitabile T, Nowomiejska K, Gagliano C, Tripodi S, et al. Five-year follow-up of secondary iris-claw intraocular lens implantation for the treatment of aphakia: anterior chamber versus retropupillary implantation. PLoS ONE. 2019;14(4):e214140.

33. Tourino Peralba R, Lamas-Francis D, Sarandeses-Diez T, Martinez-Perez L, Rodriguez-Ares T. Iris-claw intraocular lens for aphakia: can location influence the final outcomes? J Cataract Refract Surg. 2018;44(7):818-26.

34. Kristianslund O, Raen M, Ostern AE, Drolsum L. Late inthe-bag Intraocular lens dislocation: a randomized clinical trial comparing lens repositioning and lens exchange. Ophthalmology. 2017;124(2):151-9.

35. Baykara M, Ozcetin H, Yilmaz S, Timucin OB. Posterior iris fixation of the iris-claw intraocular lens implantation through a scleral tunnel incision. Am J Ophthalmol. 2007;144(4):586-91.

36. Hayashi K, Hirata A, Hayashi H. Possible predisposing factors for in-the-bag and out-of-the-bag intraocular lens dislocation and outcomes of intraocular lens exchange surgery. Ophthalmology. 2007;114(5):969-75.

37. Jakobsson G, Zetterberg M, Sundelin K, Stenevi U. Surgical repositioning of intraocular lenses after late dislocation: complications, effect on intraocular pressure, and visual outcomes. JCataract RefractSurg. 2013;39(12):1879-85.

38. Malbran ES, Malbran E Jr., Negri I. Lens guide suture for transport and fixation in secondary IOL implantation after intracapsular extraction. Int Ophthalmol. 1986;9(2-3):151-60.

39. Lewis JS. Ab externo sulcus fixation. Ophthalmic Surg. 1991;22(11):692-5.

40. Cavallini GM, Volante V, De Maria M, Torlai G, Forlini M, Chiesi L, et al. Long-term analysis of IOL stability of the Lewis technique for scleral fixation. Eur J Ophthalmol. 2015;25(6):525-8.

41. Khan MA, Gupta OP, Smith RG, Ayres BD, Raber IM, Bailey RS, et al. Scleral fixation of intraocular lenses using Gore-Tex suture: clinical outcomes and safety profile. Br J Ophthalmol.2016;100(5):638-43.

42. Hayashi K, Hayashi H, Nakao F, Hayashi F. Intraocular lens tilt and decentration, anterior chamber depth, and refractive error after trans-scleral suture fixation surgery. Ophthalmology. 1999;106(5):878-82.

43. Kemer Atik B, Altan C, Agca A, Kirmaci A, Yildirim Y, Genc S, et al. The effect of intraocular lens tilt on visual outcomes in scleral-fixated intraocular lens implantation. Int Ophthalmol.2020;40(3):717-24.

44. Price MO, Price FW Jr., Werner L, Berlie C, Mamalis N. Late dislocation of scleral-sutured posterior chamber intraocular lenses. JCataract Refract Surg. 2005;31(7):1320-6.

45. Kokame GT, Yanagihara RT, Shantha JG, Kaneko KN. Longterm outcome of pars plana vitrectomy and sutured scleralfixated posterior chamber Intraocular lens implantation or repositioning. Am J Ophthalmol. 2018;189:10-6.

46. Malta JB, Banitt M, Musch DC, Sugar A, Mian SI, Soong HK. Long-term outcome of combined penetrating keratoplasty with scleral-sutured posterior chamber intraocular lens implantation. Cornea.2009;28(7):741-6.

47. Assia EI, Nemet A, Sachs D. Bilateral spontaneous subluxation of scleral-fixated intraocular lenses. J Cataract Refract Surg. 2002;28(12):2214-6.
48. Szurman P, Petermeier K, Aisenbrey S, Spitzer MS, Jaissle GB. Z-suture: a new knotless technique for transscleral suture fixation of intraocular implants. Br J Ophthalmol. 2010;94(2):167-9.

49. Lanzetta P, Bandello FM, Virgili G, Crovato S, Menchini U. Is scleral fixation a safe procedure for intraocular lens implantation? Doc Ophthalmol. 1999;97(3-4):317-24.

50. LukAS, Young AL, Cheng LL. Long-term outcome of scleralfixated intraocular lens implantation. Br J Ophthalmol. 2013;97(10):1308-11.

51. Dalby M, Kristianslund O, Drolsum L. Long-term outcomes after surgery for late in-the-bag Intraocular lens dislocation: a randomized clinical trial. Am J Ophthalmol. 2019;207:184-94.

52. Gabor SG, Pavlidis MM. Sutureless intrascleral posterior chamber intraocular lens fixation. J Cataract Refract Surg. 2007;33(11):1851-4.

53. Scharioth GB, Prasad S, Georgalas I, Tataru C, Pavlidis M. Intermediate results of sutureless intrascleral posterior chamber intraocular lens fixation. J Cataract Refract Surg. 2010;36(2):254-9.

54. Agarwal A, Kumar DA, Jacob S, Baid C, Agarwal A, Srinivasan $S$. Fibrin glue-assisted sutureless posterior chamber intraocular lens implantation in eyes with deficient posterior capsules. J Cataract Refract Surg. 2008;34(9):1433-8.

55. Kumar DA, Agarwal A, Agarwal A, Chandrasekar R, Priyanka V. Long-term assessment of tilt of glued intraocular lenses: an optical coherence tomography analysis 5 years after surgery. Ophthalmology. 2015;122(1):48-55.

56. Prenner JL, Feiner L, Wheatley HM, Connors D. A novel approachfor posterior chamberintraocularlens placement or rescue via a sutureless scleral fixation technique. Retina. 2012;32(4):853-5.

57. Todorich B, Thanos A, Woodward MA, Wolfe JD. Sutureless intrascleral fixation of secondary intraocular lens using 27gauge vitrectomy system. Ophthalmic Surg Lasers Imaging Retina. 2016;47(4):376-9.

58. Totan Y, Karadag R. Trocar-assisted sutureless intrascleral posterior chamber foldable intra-ocular lens fixation. Eye (Lond). 2012;26(6):788-91.

59. Yamane S, Inoue M, Arakawa A, Kadonosono K. Sutureless 27-gauge needle-guided intrascleral intraocular lens implantation with lamellar scleral dissection. Ophthalmology. 2014;121(1):61-6.

60. Yamane S, Sato S, Maruyama-Inoue M, Kadonosono K. Flanged intrascleral intraocular lens fixation with doubleneedle technique. Ophthalmology. 2017;124(8):1136-42.

61. Mizuno Y, Sugimoto Y. A comparative study of transscleral suture-fixated and scleral-fixated intraocular lens implantation. Int Ophthalmol.2019;39(4):839-45.

62. Bonnell AC, Mantopoulos D, Wheatley HM, Prenner JL. Surgical technique for sutureless intrascleral fixation of a 3-piece intraocular lens using a 30-gauge needle. Retina. 2019;39(Suppl 1):S13-S5.

63. Canabrava S, Bernardino L, Batisteli T, Lopes G, DinizFilho A. Double-flanged-haptic and capsular tension ring or segment for sutureless fixation in zonular instability. Int Ophthalmol.2018;38(6):2653-62.

64. Canabrava S, Canedo Domingos Lima AC, Ribeiro G. Fourflanged Intrascleral Intraocular lens fixation technique: no flaps, no knots, no glue. Cornea. 2020;39(4):527-8.

65. Barca Francesco MD, Caporossi Tomaso MD, Angelis Lorenzo MD de; Giansanti Fabrizio MD, Savastano Alfonso MD, DiLeo Laura MD, RizzoStanislao MD. JCataractRefract Surg. 2020;46(5):716-720

66. Ganekal S, Venkataratnam S, Dorairaj S, Jhanji V. Comparative evaluation of suture-assisted and fibrin glue-assisted 
scleral fixated intraocular lens implantation. J Refract Surg. 2012;28(4):249-52.

Hinweis des Verlags Der Verlag bleibt in Hinblick auf geografische Zuordnungen und Gebietsbezeichnungen in veröffentlichten Karten und Institutsadressen neutral. 